\title{
Violating the thermodynamic uncertainty relation in the three-level maser
}

\author{
Alex Arash Sand Kalaee, ${ }^{1}$ Andreas Wacker $\odot,{ }^{1}$ and Patrick P. Potts $\odot^{1,2}$ \\ ${ }^{1}$ Mathematical Physics and NanoLund, Lund University, Box 118, 22100 Lund, Sweden \\ ${ }^{2}$ Department of Physics, University of Basel, Klingelbergstrasse 82, 4056 Basel, Switzerland
}

(Received 13 March 2021; accepted 29 June 2021; published 26 July 2021)

\begin{abstract}
Nanoscale heat engines are subject to large fluctuations which affect their precision. The thermodynamic uncertainty relation (TUR) provides a trade-off between output power, fluctuations, and entropic cost. This tradeoff may be overcome by systems exhibiting quantum coherence. This Letter provides a study of the TUR in a prototypical quantum heat engine, the Scovil-Schulz-DuBois maser. Comparison with a classical reference system allows us to determine the effect of quantum coherence on the performance of the heat engine. We identify analytically regions where coherence suppresses fluctuations, implying a quantum advantage, as well as regions where fluctuations are enhanced by coherence. This quantum effect cannot be anticipated from the off-diagonal elements of the density matrix. Because the fluctuations are not encoded in the steady state alone, TUR violations are a consequence of coherence that goes beyond steady-state coherence. While the system violates the conventional TUR, it adheres to a recent formulation of a quantum TUR. We further show that parameters where the engine operates close to the conventional limit are prevalent and TUR violations in the quantum model are not uncommon.
\end{abstract}

DOI: 10.1103/PhysRevE.104.L012103

Introduction. Nanoscale heat engines [1] have become a topic of wide interest in recent years. In such devices quantum effects become relevant and radically alter the dynamical and thermodynamic properties [2-8]. Nanoscale systems are subject to strong fluctuations in the output power $P$ which become important when quantifying useful properties such as efficiency and precision $[9,10]$. At the same time, operation of any engine is associated with the entropy production rate $\sigma$, which quantifies the thermodynamic cost. It is desirable for an engine to have both a low entropy production rate and high precision. Their trade-off can be quantified by the dimensionless thermodynamic uncertainty

$$
\mathcal{Q}=\frac{\sigma}{k_{B}} \frac{\operatorname{var}(P)}{\langle P\rangle^{2}},
$$

where $\langle P\rangle$ and $\operatorname{var}(P)$ denote the mean and variance of the power in the long time limit and $k_{B}$ is the Boltzmann constant. The thermodynamic uncertainty relation (TUR) provides a lower bound, $\mathcal{Q} \geqslant 2$ [11-17], which highlights the fundamental relevance of the thermodynamic uncertainty $\mathcal{Q}$.

The TUR has been applied to biomolecular processes $[11,13,18,19]$, heat transport [20], and Brownian clocks [21]. Furthermore, experimental realizations are an area of active development [22-26]. Generalizations have been formulated to cover discrete or time-dependent driving [21,27-30], underdamped Langevin dynamics [31-33], and the presence

Published by the American Physical Society under the terms of the Creative Commons Attribution 4.0 International license. Further distribution of this work must maintain attribution to the author(s) and the published article's title, journal citation, and DOI. Funded by Bibsam. of measurement and feedback [34]. Beyond the classical regime, quantum effects influence work, fluctuations, and entropy production [35-37], altering the TUR bounds on quantum stochastic systems [25,38-42], e.g., due to coherences [43-46] or particle exchange correlations [20,38,47]. Understanding the detailed reasons for such TUR violations and the possible establishment of more general bounds are important questions in ongoing research.

The Scovil-Schulz-DuBois (SSDB) three-level maser is the prototype for quantum heat engines relying on quantum coherence to do work [48]. In this work we study the thermodynamic uncertainty in the SSDB maser in detail, finding TUR violations induced by coherence, in analogy to Ref. [43]. A comparison to a classical model which obeys the TUR allows us to identify regions of operation where quantum dynamics results in improved operation as quantified by a lower value of $\mathcal{Q}$. Interestingly, such a quantum advantage cannot be anticipated from the off-diagonal elements of the density matrix because the fluctuations are not encoded in the steady state alone. TUR violations should thus be seen as a consequence of coherent dynamics going beyond steady-state coherence.

Quantum advantage in the maser. Figure 1 shows the maser [48], where the three levels are the lower $l$ and upper $u$ lasing levels and the excited state $x$. If $n_{l}>n_{u}$, where $n_{\alpha}$ is the relevant occupation of the Bosonic bath coupled to level $\alpha$ with energy $\omega_{\alpha}$, we obtain inversion between the laser levels, resulting in maser operation. The steady-state work output of this type of engine is well known from earlier analysis in interaction with classical [49-53] or quantized [54] light.

We probe the thermodynamic uncertainty of the SSDB maser $\mathcal{Q}$ and compare it with the uncertainty of an equivalent classical system $\mathcal{Q}^{\mathrm{cl}}$, where the coherent transition between $u$ and $l$ is replaced by a classical rate (see Fig. 2). 

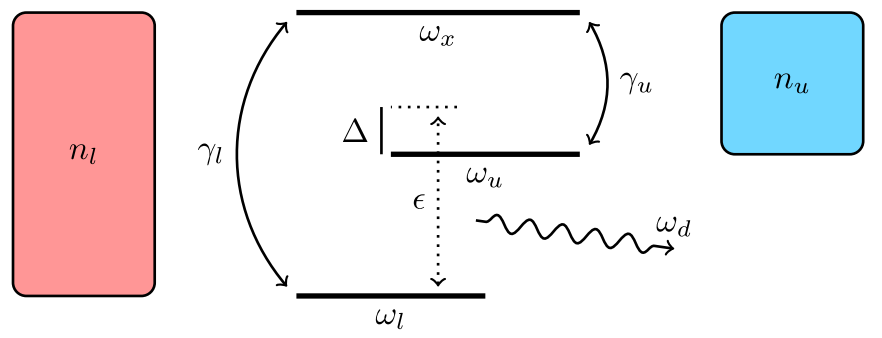

FIG. 1. Illustration of the three-level maser with energies $\omega_{\alpha}$ for $\alpha \in\{x, u, l\}$. Bath $\alpha$ with population $n_{\alpha}$ induces a transition with rate $\gamma_{\alpha}$. The external ac field connects the levels $u$ and $l$ with strength $\epsilon$ and frequency $\omega_{d}=\Delta+\omega_{u}-\omega_{l}$. In one cycle of operation, the system is excited from bath $l$ to an excited state $x$, from which it relaxes to the upper laser level $u$ by emitting heat to bath $u$. The cycle is closed by a photon that is emitted into the driving field, producing work.

While the classical system always adheres the TUR, we find regions where $\mathcal{Q}$ can go as low as 1.68 , a significant violation enabled by the quantum-coherent dynamics of the maser. These violations occur at intermediate driving strength $\epsilon$ [see Fig. 2(a)], while the classical model captures the behavior of the quantum model for both weak and strong driving. For small $\epsilon$ a perturbative treatment of the drive is justified, while for large $\epsilon$, the statistics is determined by the rates which mediate heat transfer with the baths, $\gamma_{l}$ and $\gamma_{u}$. Figure 2(b) shows that the most significant TUR violation occurs at low population $n_{u}$, where the associated temperature and decoherence are low. Interestingly, coherence may also be disadvantageous, i.e., $\mathcal{Q}>\mathcal{Q}^{\text {cl }}$. This disadvantage happens at finite detuning $|\Delta|>\Gamma$, where $\Gamma=\left(\gamma_{u} n_{u}+\gamma_{l} n_{l}\right) / 2$ denotes the broadening of the transition [which is equal to the decoherence rate; see Fig. 2(c)].

Recently, it was suggested that quantum systems which evolve according to Lindblad-type master equations adhere to a quantum TUR [41] $\mathcal{Q} \geqslant \mathcal{B}$ (see [55] for a detailed evaluation of $\mathcal{B}$ for the SSDB). For the parameters which provided a significant violation of the classical TUR we find that the SSDB maser remains well within adherence to the quantum TUR [see Figs. 2(a)-2(c)].

The system master equation. For a quantitative description, the system is assumed to have a Markovian time evolution governed by the Lindblad master equation [56] (we use $\hbar=$ $k_{B}=1$ in the following),

$\dot{\rho}=-i[H(t), \rho]+\sum_{\alpha}\left\{\gamma_{\alpha}\left(n_{\alpha}+1\right) \mathcal{D}_{\sigma_{\alpha x}}[\rho]+\gamma_{\alpha} n_{\alpha} \mathcal{D}_{\sigma_{x \alpha}}[\rho]\right\}$,

with the dissipator $\mathcal{D}_{\sigma}[\rho]=\sigma \rho \sigma^{\dagger}-\frac{1}{2}\left\{\sigma^{\dagger} \sigma \rho+\rho \sigma^{\dagger} \sigma\right\}$. Here we introduced the bath populations $n_{\alpha}$ and the respective transition rates $\gamma_{\alpha}$ for $\alpha \in\{u, l\}$. The Hamiltonian $H(t)=H_{0}+$ $V(t)$ consists of a bare term $H_{0}=\omega_{l} \sigma_{l l}+\omega_{u} \sigma_{u u}+\omega_{x} \sigma_{x x}$ and an external classical field $V(t)=\epsilon\left(e^{i \omega_{d} t} \sigma_{l u}+e^{-i \omega_{d} t} \sigma_{u l}\right)$ with frequency $\omega_{d}$ and strength $\epsilon$. The transition operators are defined as $\sigma_{i j}=|i\rangle\langle j|$.

In addition to the quantum SSDB maser we also consider a classical reference system (with a superscript cl) described by the master equation

$$
\begin{aligned}
\dot{\rho}^{\mathrm{cl}}= & \gamma_{c}\left(\mathcal{D}_{\sigma_{u l}}\left[\rho^{\mathrm{cl}}\right]+\mathcal{D}_{\sigma_{l u}}\left[\rho^{\mathrm{cl}}\right]\right)+\sum_{\alpha}\left\{\gamma_{\alpha}\left(n_{\alpha}+1\right) \mathcal{D}_{\sigma_{\alpha x}}\left[\rho^{\mathrm{cl}}\right]\right. \\
& \left.+\gamma_{\alpha} n_{\alpha} \mathcal{D}_{\sigma_{x \alpha}}\left[\rho^{\mathrm{cl}}\right]\right\}
\end{aligned}
$$

where we have introduced the classical transition rate

$$
\gamma_{c}=\frac{2 \epsilon^{2} \Gamma}{\Delta^{2}+\Gamma^{2}}
$$

as obtained by Fermi's golden rule assuming Lorentzian broadening, which provides the same work output as the quantum model.

The quantities of interest. In order to calculate $\mathcal{Q}$ we need the mean and variance of the power as well as the entropy production rate. Let $N$ denote the number of cycles done in the nominal direction (i.e., $l \rightarrow x \rightarrow u \rightarrow l$ ) minus the number of cycles in opposite direction. In each cycle, a photon is exchanged with each heat bath. As shown in the Supplemental Material [55], we further find $\langle P\rangle=\omega_{d}\langle\dot{N}\rangle$ and $\operatorname{var}(P)=$ $\omega_{d}^{2} \operatorname{var}(\dot{N})$, consistent with the picture that a single photon is emitted into the drive field per cycle. We note that this simple relation between heat and work was observed before [57] and that it may break down for higher cumulants [58].

The entropy production rate reads [59]

$$
\sigma=-\frac{\dot{Q}_{u}}{T_{u}}-\frac{\dot{Q}_{l}}{T_{l}}
$$

where $\dot{Q}_{\alpha}$ is the net rate of heat transferred from bath $\alpha$ to the engine. $T_{\alpha}$ is the bath temperature defined from the BoseEinstein distribution

$$
T_{\alpha}=\frac{\Omega_{\alpha}}{\ln \left(1+\frac{1}{n_{\alpha}}\right)},
$$

where $\Omega_{\alpha}=\left|\dot{Q}_{\alpha} /\langle\dot{N}\rangle\right|$ denotes the heat per particle transfer. We note that by using Eq. (6), our results hold for both the definition of heat and work via the full or bare Hamiltonian; see Ref. [53] for more information on this debate. This yields a positive definite entropy production rate

$$
\sigma=\ln \left[\frac{n_{l}\left(n_{u}+1\right)}{n_{u}\left(n_{l}+1\right)}\right]\langle\dot{N}\rangle>0,
$$

as $\langle\dot{N}\rangle$ has the same sign as the thermodynamic driving $n_{l}-n_{u}$ [see Eq. (9)]. Inserting the power and entropy into the definition of $\mathcal{Q}$, we obtain

$$
\mathcal{Q}=\ln \left[\frac{n_{l}\left(n_{u}+1\right)}{n_{u}\left(n_{l}+1\right)}\right] F,
$$

where we introduced the Fano factor $F=\frac{\operatorname{var}(\dot{N})}{\langle\dot{N}\rangle}$.

To determine the mean and variance of $\dot{N}$ we employ full counting statistics [60-62], in which we modify the master equations by introducing counting fields to keep track of the number of energy quanta exchanged with the baths. This procedure yields the same mean rate for the quantum and classical models as detailed in the Supplemental Material [55],

$$
\langle\dot{N}\rangle=\frac{\gamma_{c} \gamma_{u} \gamma_{l}\left(n_{l}-n_{u}\right)}{\gamma_{u} \gamma_{l}\left(3 n_{l} n_{u}+n_{u}+n_{l}\right)+2 \gamma_{c}\left(3 \Gamma+\gamma_{u}+\gamma_{l}\right)} .
$$


(a)

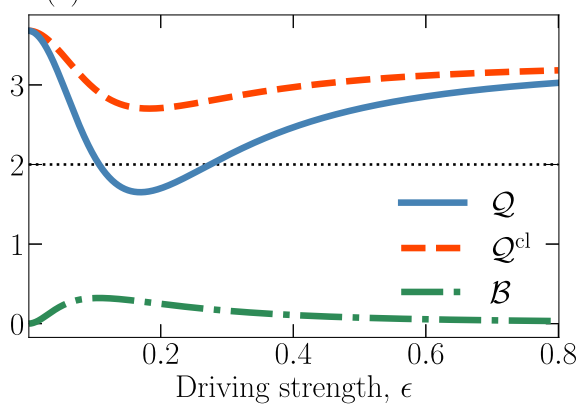

(b)

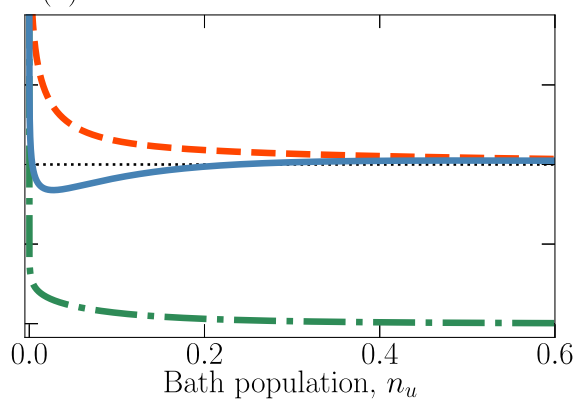

(c)

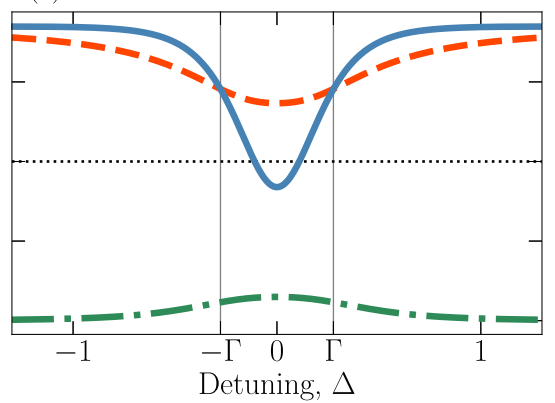

FIG. 2. Probe of thermodynamic uncertainty for the SSDB maser $\mathcal{Q}$ (solid blue line) and the classical reference system $\mathcal{Q}^{\mathrm{cl}}$ (dashed orange line) along (a) driving strength $\epsilon$, (b) bath population $n_{u}$, and (c) detuning $\Delta$. The fixed parameters are $\gamma_{u}=2, \gamma_{l}=0.1$, and $n_{l}=5$, while the parameters $n_{u}=0.027, \epsilon=0.15$, and $\Delta=0$ are varied according to the respective panels. The classical TUR limit is indicated by the dotted black line, and the quantum TUR bound $\mathcal{B}$ is indicated by the dash-dotted green line.

The Fano factor can be written as

$$
F=\frac{n_{l}\left(n_{u}+1\right)+n_{u}\left(n_{l}+1\right)}{n_{l}-n_{u}}-2\langle\dot{N}\rangle C,
$$

where $C$ takes the values

$$
\begin{aligned}
C^{\mathrm{cl}} & =\frac{2 \gamma_{c}+4 \Gamma+\gamma_{l}+\gamma_{u}}{D}, \\
C & =C^{\mathrm{cl}}+\frac{\Gamma^{2}-\Delta^{2}}{\Delta^{2}+\Gamma^{2}} \frac{\gamma_{u} \gamma_{l}}{\Gamma} \frac{\left(3 n_{l} n_{u}+n_{l}+n_{u}\right)}{D},
\end{aligned}
$$

with $D=\gamma_{u} \gamma_{l}\left(3 n_{l} n_{u}+n_{u}+n_{l}\right)+2 \gamma_{c}\left(3 \Gamma+\gamma_{u}+\gamma_{l}\right)$, for the classical and quantum models, respectively. In contrast to the average rate, the variance thus differs between the quantum and classical models. Here $C$ is larger (smaller) than $C^{\mathrm{cl}}$ if the detuning $|\Delta|$ is smaller (larger) than the broadening $\Gamma$. From Eqs. (8) and (10) we find

$$
\mathcal{Q}-\mathcal{Q}^{\mathrm{cl}}=2\langle\dot{N}\rangle \ln \left[\frac{n_{l}\left(n_{u}+1\right)}{n_{u}\left(n_{l}+1\right)}\right]\left(C^{\mathrm{cl}}-C\right) .
$$

We find the quantum model results in reduced noise $F$ and thermodynamic uncertainty $\mathcal{Q}$ for small detuning $|\Delta|<\Gamma$. This result establishes the condition for a quantum advantage in the SSDB engine, implying that this engine outperforms its classical limit. Furthermore, when $\mathcal{Q}<2$, the SSDB engine outperforms all classical engines where the TUR holds, which includes all Markovian classical engines. We note that there may exist non-Markovian classical engines which outperform the SSDB engine.

Monte Carlo exploration. To explore the thermodynamic behavior of the model we systematically evaluated $\mathcal{Q}$ by Monte Carlo sampling over a region of the parameter space (see Fig. 3). Both the quantum and classical models operate close to the conventional TUR limit $\mathcal{Q}=2$ in the great majority of sampled operation points. Quantum violations of the conventional TUR are not uncommon [see the inset in Fig. 3(a)]. On the other hand, $\mathcal{Q}^{\mathrm{cl}} \geqslant 2$ is always satisfied, as expected for systems based on classical rate equations. The range of $\mathcal{Q}$ stretches slightly wider to both smaller and larger values than $\mathcal{Q}^{\text {cl }}$ due to the possibility of both quantum advantage and disadvantage [see Figs. 3(a) and 3(b)].
Bounds for $\mathcal{Q}^{\mathrm{cl}}$. Based on Eqs. (8) and (10), the thermodynamic uncertainty can be cast into

$$
\mathcal{Q}=\mathcal{Q}_{\text {pop }}+\mathcal{Q}_{\text {tr }}
$$

where the first (population) term depends only on the bath populations and is the same for the quantum and classical models,

$$
\mathcal{Q}_{\text {pop }}=\ln \left[\frac{n_{l}\left(n_{u}+1\right)}{n_{u}\left(n_{l}+1\right)}\right] \frac{n_{l}\left(n_{u}+1\right)+n_{u}\left(n_{l}+1\right)}{n_{l}-n_{u}} .
$$

This term always adheres to the TUR, i.e., $\mathcal{Q}_{\text {pop }} \geqslant 2$ [55]. The transport term, which is essential for TUR violations, is given
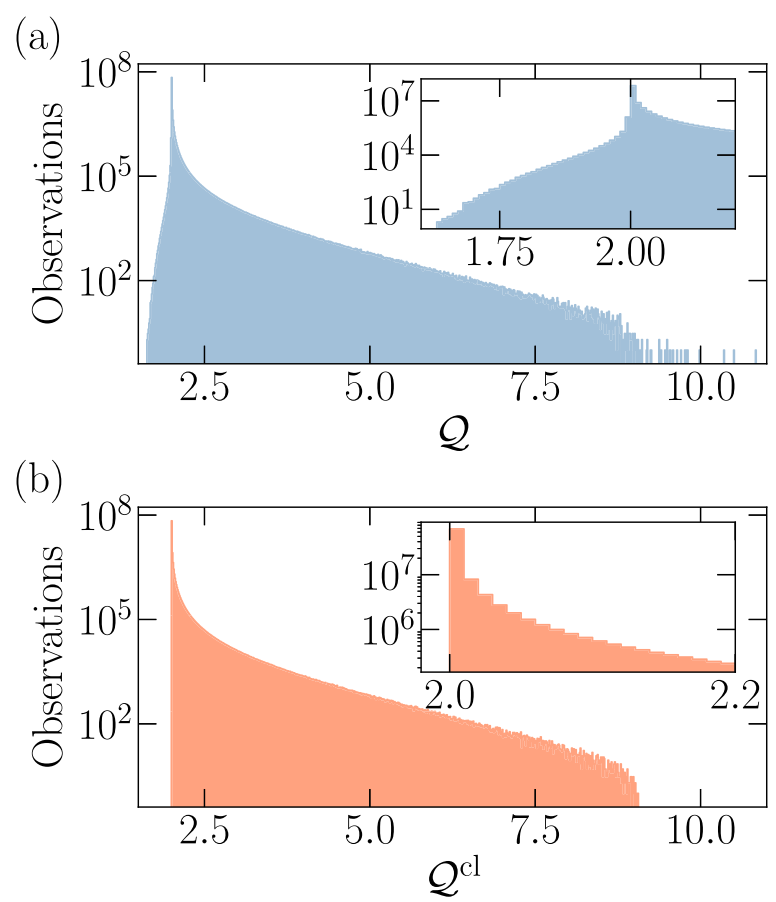

FIG. 3. Histograms of sampled values of (a) $\mathcal{Q}$ and (b) $\mathcal{Q}^{\text {cl }}$ from Monte Carlo exploration of the parameter space. Insets show the subset of the sampled data in the vicinity of the classical TUR limit. The parameters are sampled from the uniform distributions $\gamma_{\alpha} \in\left[10^{-4} ; 5\right], n_{\alpha} \in\left[10^{-4} ; 10\right], \epsilon \in\left[10^{-4} ; 1\right]$, and $\Delta \in[0 ; 1]$, and the $10^{8}$ data points are arranged in bins with a width of 0.01 . 

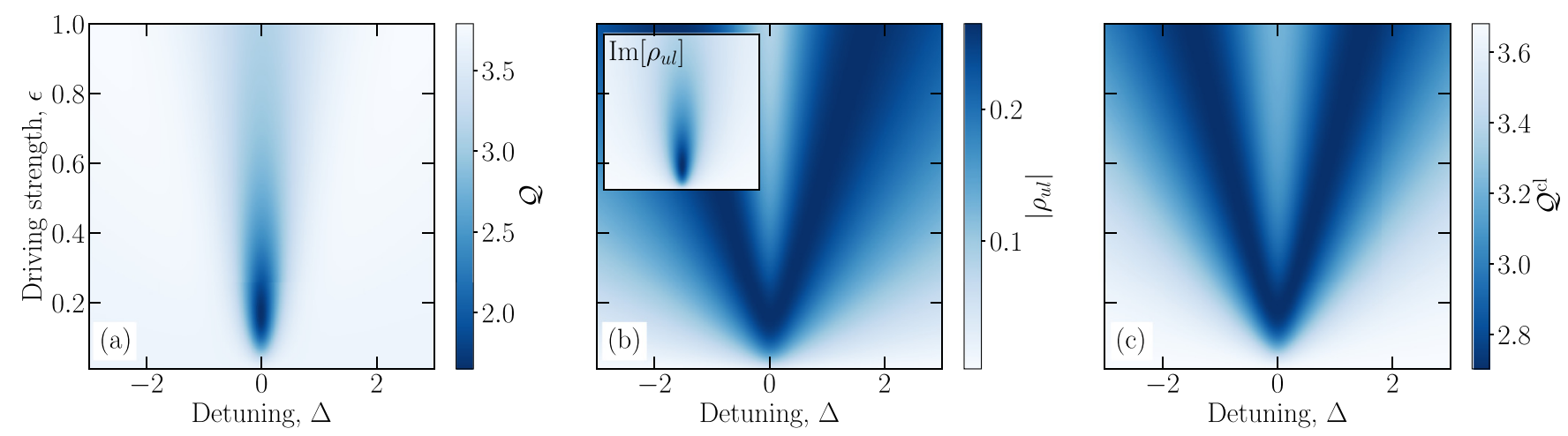

FIG. 4. Heat maps of (a) $\mathcal{Q}$ for the SSDB maser, (b) the off-diagonal matrix element $\left|\rho_{u l}\right|$ and its imaginary component (inset), and (c) $\mathcal{Q}^{c l}$ for the classical reference system. The maser parameters are $\gamma_{u}=2, n_{u}=0.027, \gamma_{l}=0.1$, and $n_{l}=5$. While $\mathcal{Q}$ shows a behavior similar to $\operatorname{Im}\left[\rho_{u l}\right], \mathcal{Q}^{\mathrm{cl}}$ shows behavior similar to $\left|\rho_{u l}\right|$.

as

$$
\mathcal{Q}_{\mathrm{tr}}=-\ln \left[\frac{n_{l}\left(n_{u}+1\right)}{n_{u}\left(n_{l}+1\right)}\right] 2\langle\dot{N}\rangle C .
$$

In the classical case, Eq. (11) provides $C^{\mathrm{cl}}>0$, and the transport term is negative. As TUR is always satisfied here, we have the restriction $2 \leqslant \mathcal{Q}^{\text {cl }} \leqslant \mathcal{Q}_{\text {pop }}$. Both inequalities fail in the quantum case, as we have the quantum advantage $C>C^{\mathrm{cl}}$ for $|\Delta|<\Gamma$, and $C$ can actually become negative for large values of $|\Delta|$. In agreement with Fig. 3, the thermodynamic uncertainty may thus take on a larger range of values in the quantum case.

Quantum coherence and TUR. Previous work on TUR in quantum systems showed that quantum coherence can be responsible for TUR violations [43,44,46]. In Fig. 4 we compare $\mathcal{Q}$ with the steady-state coherence $\rho_{u l}=\langle u|\rho| l\rangle$ between the lasing levels as a function of detuning and driving. We find particularly low values of $\mathcal{Q}$ for zero detuning and moderate driving in Fig. 4(a). This pattern differs entirely from the coherence $\left|\rho_{u l}\right|$ shown in Fig. 4(b), which exhibits a V-shaped ridge [55]. Surprisingly, a similar ridge is found in $\mathcal{Q}^{\text {cl }}$ [see Fig. 4(c)], which indicates that the absolute value $\left|\rho_{u l}\right|$ cannot be directly related to quantum effects in $\mathcal{Q}$. Furthermore, the $\mathrm{V}$-shaped coherence pattern extends into regions $|\Delta|>$ $\Gamma$, where quantum effects provide $\mathcal{Q}>\mathcal{Q}^{\text {cl }}$, i.e., a quantum disadvantage.

On the other hand, the inset in Fig. 4(b) shows that $\operatorname{Im}\left[\rho_{u l}\right]$ might be a candidate to explain the TUR violations. In this context, we note that

$$
\langle\dot{N}\rangle=2 \epsilon \operatorname{Im}\left[\rho_{u l}\right],
$$

as can be seen from the relation $\langle P\rangle=-\operatorname{Tr}\left[\left(\partial_{t} H\right) \rho\right]$. Nevertheless, a finite rate does not ensure the presence of coherence, as the classical model produces the exact same amount of power. Equation (12) shows that any quantum advantage is proportional to $\langle\dot{N}\rangle$. Thus, it is rather the mean rate, which can be reproduced by the classical model, than the coherence itself, which is of relevance here.
What distinguishes TUR in the quantum system from its classical counterpart is the variance, a quantity that goes beyond what is encoded in the steady state. Hence, the steady-state coherence cannot fully capture what sets quantum-coherent dynamics apart from the classical system. A similar conclusion was reached in Ref. [63].

Conclusion. In this Letter we studied the performance of the Scovil-Schulz-DuBois heat engine in terms of the thermodynamic uncertainty $\mathcal{Q}$. From the analytical expressions we find that the quantum-coherent dynamics exhibits a quantum advantage compared to its classical counterpart if the detuning is less than the broadening, $|\Delta|<\Gamma$. In cases of great quantum advantage, the maser violates the conventional TUR limit $\mathcal{Q}>2$. Parameter points where the engine operates close to the conventional limit are prevalent, and violations are not uncommon. We also showed that this engine adheres to a recent formulation of a quantum TUR.

While quantum advantages and disadvantages over classical systems are related to the quantum-coherent dynamics, we cannot establish a direct relation between the behavior of the steady-state coherence and that of the thermodynamic uncertainty. The thermodynamic uncertainty depends on the variance of the output power, whose properties, unlike the mean, are not fully imprinted onto the state of the system. This illustrates a need to introduce new quantifiers that measure the coherence present in the dynamics of a system going beyond the density matrix.

Acknowledgments. We thank the Knut and Alice Wallenberg Foundation (Project No. 2016.0089) and NanoLund for financial support. P.P.P. acknowledges funding from the European Union's Horizon 2020 research and innovation program under Marie Skłodowska-Curie Grant Agreement No. 796700, from the Swedish Research Council (Starting Grant No. 2020-03362), and from the Swiss National Science Foundation (Eccellenza Professorial Fellowship PCEFP2_194268).
[1] G. Benenti, G. Casati, K. Saito, and R. S. Whitney, Fundamental aspects of steady-state conversion of heat to work at the nanoscale, Phys. Rep. 694, 1 (2017).
[2] B. Sothmann, R. Sánchez, and A. N. Jordan, Thermoelectric energy harvesting with quantum dots, Nanotechnology 26, 032001 (2014). 
[3] J. Millen and A. Xuereb, Perspective on quantum thermodynamics, New J. Phys. 18, 011002 (2016).

[4] S. Vinjanampathy and J. Anders, Quantum thermodynamics, Contemp. Phys. 57, 545 (2016).

[5] A. Streltsov, G. Adesso, and M. B. Plenio, Colloquium: Quantum coherence as a resource, Rev. Mod. Phys. 89, 041003 (2017).

[6] M. Josefsson, A. Svilans, A. M. Burke, E. A. Hoffmann, S. Fahlvik, C. Thelander, M. Leijnse, and H. Linke, A quantumdot heat engine operating close to the thermodynamic efficiency limits, Nat. Nanotechnol. 13, 920 (2018).

[7] F. Binder, L. A. Correa, C. Gogolin, J. Anders, and G. Adesso, Thermodynamics in the Quantum Regime: Fundamental Aspects and New Directions (Springer, Cham, 2018).

[8] P. Talkner and P. Hänggi, Colloquium: Statistical mechanics and thermodynamics at strong coupling: Quantum and classical, Rev. Mod. Phys. 92, 041002 (2020).

[9] U. Seifert, Stochastic thermodynamics, fluctuation theorems and molecular machines, Rep. Prog. Phys. 75, 126001 (2012).

[10] G. Verley, M. Esposito, T. Willaert, and C. Van den Broeck, The unlikely Carnot efficiency, Nat. Commun. 5, 4721 (2014).

[11] A. C. Barato and U. Seifert, Thermodynamic Uncertainty Relation for Biomolecular Processes, Phys. Rev. Lett. 114, 158101 (2015).

[12] T. R. Gingrich, J. M. Horowitz, N. Perunov, and J. L. England, Dissipation Bounds all Steady-State Current Fluctuations, Phys. Rev. Lett. 116, 120601 (2016).

[13] P. Pietzonka, A. C. Barato, and U. Seifert, Universal bound on the efficiency of molecular motors, J. Stat. Mech. (2016) 124004.

[14] P. Pietzonka, F. Ritort, and U. Seifert, Finite-time generalization of the thermodynamic uncertainty relation, Phys. Rev. E 96, 012101 (2017).

[15] P. Pietzonka and U. Seifert, Universal Trade-off between Power, Efficiency, and Constancy in Steady-State Heat Engines, Phys. Rev. Lett. 120, 190602 (2018).

[16] J. M. Horowitz and T. R. Gingrich, Thermodynamic uncertainty relations constrain non-equilibrium fluctuations, Nat. Phys. 16, 15 (2019).

[17] G. Falasco, M. Esposito, and J.-C. Delvenne, Unifying thermodynamic uncertainty relations, New J. Phys. 22, 053046 (2020).

[18] M. W. Jack, N. J. López-Alamilla, and K. J. Challis, Thermodynamic uncertainty relations and molecular-scale energy conversion, Phys. Rev. E 101, 062123 (2020).

[19] Y. Song and C. Hyeon, Thermodynamic cost, speed, fluctuations, and error reduction of biological copy machines, J. Phys. Chem. Lett. 11, 3136 (2020).

[20] S. Saryal, H. M. Friedman, D. Segal, and B. K. Agarwalla, Thermodynamic uncertainty relation in thermal transport, Phys. Rev. E 100, 042101 (2019).

[21] A. C. Barato and U. Seifert, Cost and Precision of Brownian Clocks, Phys. Rev. X 6, 041053 (2016).

[22] S. K. Manikandan, S. Ghosh, A. Kundu, B. Das, V. Agrawal, D. Mitra, A. Banerjee, and S. Krishnamurthy, Quantitative analysis of non-equilibrium systems from short-time experimental data, arXiv:2102.11374.

[23] G. Paneru, S. Dutta, T. Tlusty, and H. K. Pak, Reaching and violating thermodynamic uncertainty bounds in information engines, Phys. Rev. E 102, 032126 (2020).
[24] W. Hwang and C. Hyeon, Energetic costs, precision, and transport efficiency of molecular motors, J. Phys. Chem. Lett. 9, 513 (2018).

[25] S. Pal, S. Saryal, D. Segal, T. S. Mahesh, and B. K. Agarwalla, Experimental study of the thermodynamic uncertainty relation, Phys. Rev. Res. 2, 022044(R) (2020).

[26] H. M. Friedman, B. K. Agarwalla, O. Shein-Lumbroso, O. Tal, and D. Segal, Thermodynamic uncertainty relation in atomicscale quantum conductors, Phys. Rev. B 101, 195423 (2020).

[27] K. Proesmans and C. Van den Broeck, Discrete-time thermodynamic uncertainty relation, Europhys. Lett. 119, 20001 (2017).

[28] K. Macieszczak, K. Brandner, and J. P. Garrahan, Unified Thermodynamic Uncertainty Relations in Linear Response, Phys. Rev. Lett. 121, 130601 (2018).

[29] T. Koyuk and U. Seifert, Thermodynamic Uncertainty Relation for Time-Dependent Driving, Phys. Rev. Lett. 125, 260604 (2020).

[30] L. M. Cangemi, M. Carrega, A. De Candia, V. Cataudella, G. De Filippis, M. Sassetti, and G. Benenti, Optimal energy conversion through antiadiabatic driving breaking time-reversal symmetry, Phys. Rev. Res. 3, 013237 (2021).

[31] L. P. Fischer, P. Pietzonka, and U. Seifert, Large deviation function for a driven underdamped particle in a periodic potential, Phys. Rev. E 97, 022143 (2018).

[32] J. S. Lee, J.-M. Park, and H. Park, Thermodynamic uncertainty relation for underdamped Langevin systems driven by a velocity-dependent force, Phys. Rev. E 100, 062132 (2019).

[33] T. Van Vu and Y. Hasegawa, Uncertainty relations for underdamped Langevin dynamics, Phys. Rev. E 100, 032130 (2019).

[34] P. P. Potts and P. Samuelsson, Thermodynamic uncertainty relations including measurement and feedback, Phys. Rev. E 100, 052137 (2019).

[35] G. Kießlich, P. Samuelsson, A. Wacker, and E. Schöll, Counting statistics and decoherence in coupled quantum dots, Phys. Rev. B 73, 033312 (2006).

[36] M. Scandi, H. J. D. Miller, J. Anders, and M. Perarnau-Llobet, Quantum work statistics close to equilibrium, Phys. Rev. Res. 2, 023377 (2020).

[37] C. L. Latune, I. Sinayskiy, and F. Petruccione, Negative contributions to entropy production induced by quantum coherences, Phys. Rev. A 102, 042220 (2020).

[38] K. Brandner, T. Hanazato, and K. Saito, Thermodynamic Bounds on Precision in Ballistic Multiterminal Transport, Phys. Rev. Lett. 120, 090601 (2018).

[39] F. Carollo, R. L. Jack, and J. P. Garrahan, Unraveling the Large Deviation Statistics of Markovian Open Quantum Systems, Phys. Rev. Lett. 122, 130605 (2019).

[40] G. Guarnieri, G. T. Landi, S. R. Clark, and J. Goold, Thermodynamics of precision in quantum nonequilibrium steady states, Phys. Rev. Res. 1, 033021 (2019).

[41] Y. Hasegawa, Quantum Thermodynamic Uncertainty Relation for Continuous Measurement, Phys. Rev. Lett. 125, 050601 (2020).

[42] Y. Hasegawa, Thermodynamic Uncertainty Relation for General Open Quantum Systems, Phys. Rev. Lett. 126, 010602 (2021).

[43] K. Ptaszyński, Coherence-enhanced constancy of a quantum thermoelectric generator, Phys. Rev. B 98, 085425 (2018). 
[44] B. K. Agarwalla and D. Segal, Assessing the validity of the thermodynamic uncertainty relation in quantum systems, Phys. Rev. B 98, 155438 (2018).

[45] L. M. Cangemi, V. Cataudella, G. Benenti, M. Sassetti, and G. De Filippis, Violation of thermodynamics uncertainty relations in a periodically driven work-to-work converter from weak to strong dissipation, Phys. Rev. B 102, 165418 (2020).

[46] A. Rignon-Bret, G. Guarnieri, J. Goold, and M. T. Mitchison, Thermodynamics of precision in quantum nanomachines, Phys. Rev. E 103, 012133 (2021).

[47] J. Liu and D. Segal, Thermodynamic uncertainty relation in quantum thermoelectric junctions, Phys. Rev. E 99, 062141 (2019).

[48] H. E. D. Scovil and E. O. Schulz-DuBois, Three-Level Masers as Heat Engines, Phys. Rev. Lett. 2, 262 (1959).

[49] W. E. Lamb, Theory of an optical maser, Phys. Rev. 134, A1429 (1964).

[50] E. Geva and R. Kosloff, Three-level quantum amplifier as a heat engine: A study in finite-time thermodynamics, Phys. Rev. E 49, 3903 (1994).

[51] E. Geva and R. Kosloff, The quantum heat engine and heat pump: An irreversible thermodynamic analysis of the threelevel amplifier, J. Chem. Phys. 104, 7681 (1996).

[52] E. Boukobza and D. J. Tannor, Thermodynamics of bipartite systems: Application to light-matter interactions, Phys. Rev. A 74, 063823 (2006).

[53] A. A. S. Kalaee and A. Wacker, Positivity of entropy production for the three-level maser, Phys. Rev. A 103, 012202 (2021).
[54] W. Niedenzu, M. Huber, and E. Boukobza, Concepts of work in autonomous quantum heat engines, Quantum 3, 195 (2019).

[55] See Supplemental Material at http://link.aps.org/supplemental/ 10.1103/PhysRevE.104.L012103 for calculation details on the steady state, analytical mean, and variance of the rates for the quantum and classical models and implementation of the quantum TUR.

[56] G. Lindblad, On the generators of quantum dynamical semigroups, Commun. Math. Phys. 48, 119 (1976).

[57] K. Verteletsky and K. Mølmer, Revealing the strokes of autonomous quantum heat engines with work and heat fluctuations, Phys. Rev. A 101, 010101(R) (2020).

[58] T. Kerremans, P. Samuelsson, and P. P. Potts, Probabilistically violating the first law of thermodynamics in a quantum heat engine, arXiv:2102.01395.

[59] H. Spohn, Entropy production for quantum dynamical semigroups, J. Math. Phys. 19, 1227 (1978).

[60] M. Bruderer, L. D. Contreras-Pulido, M. Thaller, L. Sironi, D. Obreschkow, and M. B. Plenio, Inverse counting statistics for stochastic and open quantum systems: The characteristic polynomial approach, New J. Phys. 16, 033030 (2014).

[61] M. Esposito, U. Harbola, and S. Mukamel, Nonequilibrium fluctuations, fluctuation theorems, and counting statistics in quantum systems, Rev. Mod. Phys. 81, 1665 (2009).

[62] G. Schaller, Open Quantum Systems Far from Equilibrium, Lecture Notes in Physics Vol. 881 (Springer, Heidelberg, 2014).

[63] I. Medina, S. V. Moreira, and F. L. Semião, Quantum versus classical transport of energy in coupled two-level systems, Phys. Rev. A 103, 052216 (2021). 